\title{
Research on Corrosion Effects on Materials in Air Pollution Condition
}

\author{
Yiwen Wang ${ }^{1, *}$ and Venda Garilli ${ }^{2}$ \\ ${ }^{1}$ School of Resources and Environment Engineering, Wuhan University of Technology, Wuhan, China, 430074 \\ ${ }^{2}$ School of Engineering, National University of Singapore, Singapore \\ * Corresponding author: 15926424120@163.com
}

\begin{abstract}
In order to find the corrosion effects on materials in air pollution condition and to investigate the corrosion behavior of structural metals, weight change and mass loss were studied. The results obtained are discussed and their plausible interpretation is attempted. The results for the modern glass samples indicate that the increasing trend of the total deposited mass of particles per surface unit and haze, demonstrates a cumulative non-linear phenomenon with an asymptotic level of saturation.
\end{abstract}

\section{Introduction}

The damage and effects caused by air pollution on humans and the environment are gradually recognized. Air pollution includes outdoor air pollution and indoor air pollution. Outdoor air pollutants include gaseous pollutants, particle pollutants etc., and indoor air pollution is caused by excessive harmful materials in indoor air, which will affect human health. The harmful materials include formaldehyde, benzene, ammonia, radioactive radon etc. In the past20/30years, haze weather has been rapidly increasing in the eastern areas of our country, and at the same time, the visibility has been getting worse significantly. Haze pollution is becoming one kind of the main air pollution in cities. And ordinary people and scientific community have paid more and more attention to it. However, it still lacks systematic and in-deep research on health risks caused by air pollution, related economic loss, indoor air pollution characteristics and pollutants.

Reports point out: The average PM10concentrations of 25cities in Yangtze River Delta Region during 2009-20015 were $52.8-112.1 \mu \mathrm{g} / \mathrm{m}^{3}$, and among them, the average PM10 concentrations of Nanjing $\left(107.3 \mu \mathrm{g} / \mathrm{m}^{3}\right)$, Xuzhou $(112.1 \mu \mathrm{g} /$ $\mathrm{m}^{3}$ ), Yancheng $\left(107.8 \mu \mathrm{g} / \mathrm{m}^{3}\right)$ and Hangzhou $\left(108.2 \mu \mathrm{g} / \mathrm{m}^{3}\right)$ were higher than the other cities of YRDR. The PM 10 concentrations in the winter were the highest, about $110.5 \mu \mathrm{g} / \mathrm{m}^{3}$, followed by spring $\left(95.9 \mu \mathrm{g} / \mathrm{m}^{3}\right)$ and summer $(85.5 \mu \mathrm{g} /$ $\left.\mathrm{m}^{3}\right)$, with the lowest in autumn $\left(78.2 \mu \mathrm{g} / \mathrm{m}^{3}\right)$. The potential health risks of respiratory system disease caused by atmospheric particulates in Xuzhou, Yancheng, Hangzhou and Nanjing were 2.11, 2.04, 2.04 and 2.02 times respectively as much as in Zhoushan. The average concentrations of $\mathrm{SO}_{2}, \mathrm{NO}_{2}$ and $\mathrm{PM} 10$ in the urban areas of Ningbo were $1.13,1.25$ and 1.41 times as much as those in suburban areas. The health risks of urban residents were higher than the residents in the suburbs. The urban residents had the health risks caused by $\mathrm{NO}_{2}$ as high as the11times the acceptable level. The concentrations of $\mathrm{PM} 10, \mathrm{NO}_{2}$ and $\mathrm{SO}_{2}\left(63.6 \mu \mathrm{g} / \mathrm{m}^{3}, 45.3 \mu \mathrm{g} / \mathrm{m}^{3}\right.$ and $27.5 \mu \mathrm{g} / \mathrm{m}^{3}$ respectively) during haze pollution period in2009inShanghai was higher than those $\left(63.6 \mu \mathrm{g} / \mathrm{m}^{3} 、 45.3 \mu \mathrm{g} / \mathrm{m}^{3}\right.$ and $27.5 \mu \mathrm{g} / \mathrm{m}^{3}$ respectively) in non-haze pollution period. And the concentrations of $\mathrm{PM} 10, \mathrm{NO}_{2}$ and $\mathrm{SO}_{2}$ were highest in winter, followed by spring and summer, and the lowest in winter. The outpatients of two large pediatric hospitals investigated have more outpatients in winter than other seasons. The number of outpatients in non-haze pollution period is larger than haze pollution period, which shows the hysteresis effect of haze pollution on local residents' health.

\section{Experiment}

The MULTI-ASSESS project was divided into seven work packages (WPs). The University of Athens has participated in WP1, WP2 and WP4. The main experimental observations were obtained in the frame of WP4, which took place during 2013-2014, and are presented here. More specifically in this work package, specimens of structural metals, glass, natural and artificial stone materials were exposed to different environmental conditions. Some of these materials were selected because they have been used in the construction of monuments, while the rest of them are often used in the construction of modern buildings. For the purpose of this project, a station was installed on the building of the Ministry of Health and Social Solidarity of Wuhan City. This building is located in central Wuhan next to the "ring road", at a crossroads. One facade of the building is facing south, whilst the other faces to the east. Some of the specimens were 
exposed on the roof, while the rest of them were exposed at specific height levels on the east facade of the building. Due to the needs of the experiment two kinds of racks were installed at this building. A wooden rack (the main rack) was installed on the roof, facing south, while a group of three aluminum racks were installed on the ground floor, the 1st, $2 \mathrm{nd}$, 4th, 5th and 7th floor (height levels of $3 \mathrm{~m}, 6 \mathrm{~m}, 9 \mathrm{~m}, 15 \mathrm{~m}, 18 \mathrm{~m}$ and $21 \mathrm{~m}$ from the ground, respectively).

The wooden rack consisted of an inclined plane, for the specimens' display under unsheltered conditions, and an aluminum box with open bottom, for the exposure of specimens under sheltered conditions. The display of the aluminum racks was under sheltered conditions and they were distributed on the east facade of the building. The exposed structural metal samples included zinc (99.99\%), copper $(\mathrm{Cu} 99 \%$, P 0.015 to $0.04 \%)$, unalloyed carbon steel $(\mathrm{C}<0.2 \%, \mathrm{P}<0.07 \%, \mathrm{~S}<0.05 \%, \mathrm{Cu}<0.07 \%)$ and bronze $(\mathrm{Cu} 81 \%$, Sn 5.8\%, $\mathrm{Pb} 6.7 \%, \mathrm{Zn} 4.5 \%$, Ni $1.6 \%$ and trace elements). Three samples of each material were exposed at an unsheltered situation on the inclined plane of the main rack for one year. All the samples had the same dimensions $(100 \times 150 \times(1-2) \mathrm{mm} 3)$. Two samples of zinc, copper and steel were exposed at each height level, mounted on the aluminium racks for one year. All the samples had the same dimensions, but different from the samples exposed on the main rack $(100 \times 50 \times(1-2) \mathrm{mm} 3)$. Two different types of glass were exposed, medieval glass ( $\mathrm{SiO} 248 \%$, $\mathrm{K} 2 \mathrm{O} 25.5 \%, \mathrm{CaO} 15 \%$, MgO 3\%, $\mathrm{Al} 2 \mathrm{O} 31.5 \%$, P2O5 4\%, Na2O 3\%, in mass percentage) and modern glass ( $\mathrm{SiO} 271.7 \%, \mathrm{Na} 2 \mathrm{O} 13.1 \%, \mathrm{CaO} 9.6 \%, \mathrm{MgO} 4.1 \%, \mathrm{~K} 2 \mathrm{O} 0.3 \%, \mathrm{Al} 2 \mathrm{O} 30.7 \%$, $\mathrm{Fe} 2 \mathrm{O} 30.1 \%$, in mass percentage). Six samples of medieval glass $(10 \times 10 \times 2 \mathrm{~mm} 3)$, mounted three on each of two glassfibre reinforced plastic plates, were exposed in the aluminum box, while four samples, mounted two on each of two of the same kind of plastic plates, were exposed at the specified levels. The duration of the exposure was 6 months for half of the specimens and one year for the other half. Modern glass samples were exposed on the main rack. Eight samples, mounted on a wooden plate, were placed on the inclined plane and another group of eight samples, mounted on an aluminum plate, was placed into the aluminum box. All specimens had the same dimensions $(100 \times 100 \times 2 \mathrm{~mm} 3)$. Every three months a sample of each group was with-drawn. During the experiment it was decided to expose the last two specimens of each group for a longer time period.

The natural stone materials included Carrara marble (Italy), Baumberger sandstone (Germany), Gotland sandstone (Sweden), Saaremaa dolomite (Estonia) and three types of Portland limestone (England) with smooth, medium and rough surfaces. In parenthesis the country of origin of each material is depicted. Figure 1 shows the physical properties of the natural stone materials. Reference concrete was used as artificial stone material, prepared in laboratory conditions, using Portland cement CEM I 32.5N, fine-middle aggregates, Water/Cement (W/C) ratio 0.7 and having: apparent density: $2294 \mathrm{kgm}-3$, open porosity: $16.8 \%$, water absorption: $5.7 \%$, saturation coefficient: 0.8 .n. All the samples had the same dimensions $(50 \times 50 \times(8 \pm 2) \mathrm{mm} 3)$. Three samples of each material, medium type for Portland limestone, were exposed as sheltered and unsheltered on the main rack, mounted on special carousels.

Half of the samples were withdrawn after one year of exposure, while the rest of the samples remained exposed for two years. In addition, two samples of each type of Portland and concrete were exposed at the specific height levels, mentioned above.

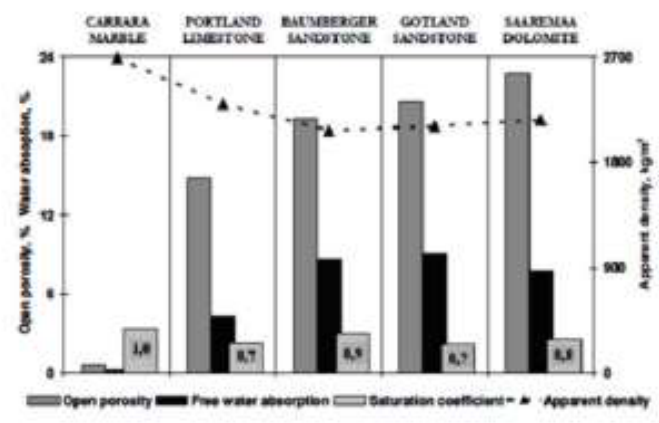

Fig. 1. Physical properties of stone materials.

\section{Results and Discussion}

In order to investigate the corrosion behavior of structural metals, weight change and mass loss were studied. Figure 2 shows the measurements obtained for the weight change of the exposed specimens at the specific height levels. It should be kept in mind that the weight change is defined as the difference between the mass of an exposed sample and its initial mass before the exposure. Obviously the weight change depends on the particular environmental conditions. In this context, under sheltered conditions, where there is no run-off, weight change is always positive, because of the uptake of material from the environment.

Under unsheltered conditions, except of the uptake process, the run-off of dissolved metal or patina components leads to a diminution of environmental compounds and weight change can be positive or negative. According to the results obtained, the unalloyed carbon steel is the most sensitive metal, among the exposed ones, to weight change, while copper is the most durable. The highest value of weight change for the steel was noticed at the level of $18 \mathrm{~m}$, while the lowest at the level of $6 \mathrm{~m}$. For zinc and copper specimens values of weight change were almost constant with 
the height. The experimental results of the mass loss, for the same specimens, are presented in Fig. 3. Keeping in mind that the mass loss is defined as mass difference between the original metal and the remaining metal after removing the corrosion layer, we reach to the conclusion that this parameter is directly correlated to the loss of metal due to run-off and compounds within the corrosion layer. The unalloyed carbon steel arises to be the most sensitive metal, among the exposed ones, to the mass loss, while copper is the most durable. For all the metals, the lowest values were noticed at the level of $6 \mathrm{~m}$ with an increase with the height.

The same parameters were also estimated for the specimens exposed as unsheltered on the main rack. The results for the weight change can be seen in Fig. 4. Unalloyed carbon steel was the material with the greatest value of weight change, while bronze was the only material with negative value of this parameter. The latter result can be explained by considering the influence of run-off on the specimens exposed under unsheltered conditions, which cause diminution of environmental compounds. Figure 5, illustrates the results for the mass loss of the same specimens as above. These results confirm that the unalloyed carbon steel is the most sensitive material to the corrosion. Zinc mass loss values were almost $70 \%$ greater than the same values of copper and bronze. Sulphate dominates among the water soluble anions, while nitrate concentrations are also high. Chloride concentrations are lower than nitrate. The water soluble anion with the lowest concentration detected is fluoride. Concentrations of nitrate, chloride and fluoride decrease with height against sulphate concentration, which increasing up to the level of $18 \mathrm{~m}$.

The most critical parameter for the specification of the weathering of medieval glass 15 specimens is the leaching depth of the constituents and especially $\mathrm{K}$ and $\mathrm{Ca}(\mathrm{d}(\mathrm{K}), \mathrm{d}(\mathrm{Ca}))$ because these elements present the most significant deterioration. After the appropriate preparation of the samples these parameters were measured using scanning electron microscopy (SEM) in combination with energy dispersive x-ray microanalysis (EDX). Mean values of the leaching depths have 20 been calculated for the specimens exposed for 6 and 12 months. According to these results, $\mathrm{K}$ is the element with the highest leaching depth.

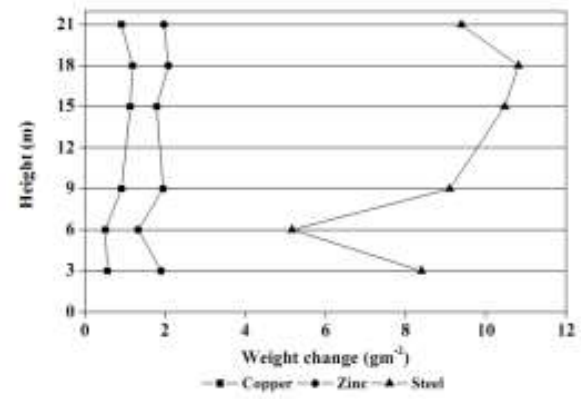

Fig. 2. Weight change of the specimens exposed

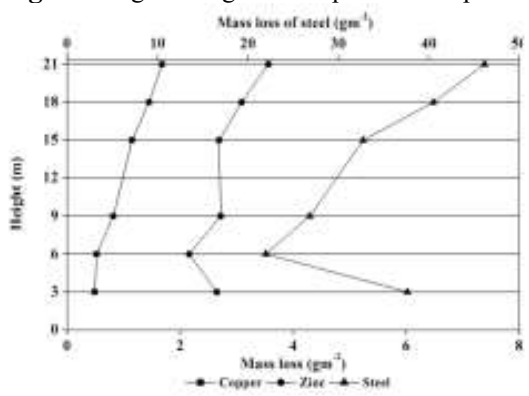

Fig. 3. Mass loss of the specimens exposed

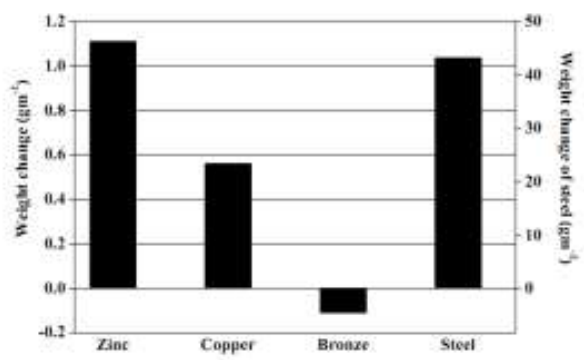

Fig. 4. Weight change of the specimens exposed for one year on the main rack (unsheltered). 


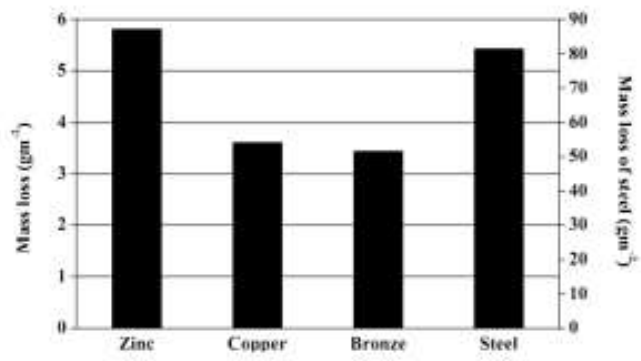

Fig. 5. Mass loss of the specimens exposed for one year on the main rack (unsheltered)

\section{References}

1. Wu He. Examining students' online interaction in a live video streaming environment using data mining and text mining. Computers in Human Behavior, (2013), pp. 291-304.

2. Fu Xiao, Cheng Fan. Data mining in building automation system for improving building operational performance. Energy \& Buildings, (2014), pp. 75-83.

3. Benrun Huang, Li Xia, Zhengguo Wu, Weiping Zhou. Control Strategy of A Dynamic Voltage Restorer (DVR) Based on Line Voltage Compensation. Energy Procedia, (2011), pp. 13-26.

4. Wei Dai, Mostafa Bassiouni. An improved task assignment scheme for Hadoop running in the clouds. Journal of Cloud Computing, (2013), pp. 21-33.

5. Y. Mishing, in: Diffusion Processes in Advanced Technological Materials, edtied by D. Gupta Noyes Publications/William Andrew Publising, Norwich, NY (2004), in press. 